\title{
CDK8 is a positive regulator of transcriptional elongation within the serum response network
}

\author{
Aaron J. Donner ${ }^{1}$, Christopher C. Ebmeier ${ }^{2}$, Dylan J. Taatjes ${ }^{2}$, and Joaquín M. Espinosa ${ }^{1}$ \\ ${ }^{1}$ Howard Hughes Medical Institute / Department of Molecular, Cellular and Developmental \\ Biology, University of Colorado at Boulder, Colorado, 80309, United States of America \\ 2 Department of Chemistry and Biochemistry, The University of Colorado at Boulder, Colorado, \\ 80309, United States of America
}

\section{Abstract}

The Mediator complex allows communication between transcription factors and RNA polymerase II (RNAPII). CDK8, the kinase found in some variants of Mediator, has been characterized mostly as a transcriptional repressor. Recently, CDK8 was demonstrated to be a potent oncoprotein. Here we show that CDK8 is a positive regulator of genes within the serum response network, including several members of the AP-1 and EGR family of oncogenic transcription factors. Mechanistic studies demonstrate that CDK8 is not required for RNAPII recruitment or promoter escape. Instead, CDK8 depletion leads to the appearance of slower elongation complexes carrying hypophosphorylated RNAPII. CDK8-Mediator regulates precise steps in the assembly of the RNAPII elongation complex, including the recruitment of P-TEFb and BRD4. Furthermore, CDK8-Mediator specifically interacts with P-TEFb. Thus, we uncovered a novel role for CDK8 in transcriptional regulation that may contribute to its oncogenic effects.

In eukaryotes, the transcription of all protein-coding genes is accomplished by a single enzyme, RNAPII. DNA-binding transcription factors and a myriad of coregulators enable activated gene transcription by RNAPII in a cell type- and signalling-specific manner. Mediator is a large protein complex found in all eukaryotes that facilitates activatordependent transcription, yet its mechanism of action is ill defined ${ }^{1}, 2$. Mediator is considered to be an integral part of the Pre-Initiation Complex (PIC), which also includes the general transcription factors (GTFs) TFIIA, TFIIB, TFIID, TFIIE, TFIIF and TFIIH ${ }^{1}{ }^{2}$. The fact that Mediator interacts directly with both transactivators and RNAPII supports the notion that it may act as a molecular bridge to facilitate activator-induced recruitment of RNAPII onto promoters ${ }^{3}{ }^{5}$. However, recent genome-wide studies demonstrate that transcriptional activation at post-RNAPII recruitment steps is a widespread phenomenon in metazoans. Paused RNAPII is found at the promoters of most genes induced by stress, developmental

Users may view, print, copy, download and text and data- mine the content in such documents, for the purposes of academic research, subject always to the full Conditions of use: http://www.nature.com/authors/editorial_policies/license.html\#terms

Correspondence: joaquin.espinosa@colorado.edu.

Authors contribution statement.

AJD performed most ChIP, Q-RT-PCR and western blots experiments, performed all nuclear run-ons and carried out microarray data analysis and wrote the manuscript. CE and DT generated all data regarding the CDK8-Mediator/P-TEFb interaction. JME performed ChIP, Q-RT-PCR and western blot experiments and carried out the microarray experiment. 
cues and various signalling events ${ }^{6}-9$. Upon stimulus, RNAPII is released from promoters and converted into an elongation competent form by poorly understood mechanisms. P$\mathrm{TEFb}$ (Positive Transcription Elongation Factor-b) is recognized as a critical elongation factor required for most RNAPII-dependent transcription ${ }^{10}$. Recruitment of P-TEFb by transactivators, the viral protein Tat or the chromatin-binding protein BRD4 stimulates elongation in a number of experimental systems ${ }^{11}{ }^{13}$. CDK9, the kinase subunit of P-TEFb, phosphorylates Ser2 of the C-terminal domain (CTD) heptad repeats (YSPTSPS) of RPB1, the largest subunit of RNAPII ${ }^{14}{ }^{16}$. CTD phosphorylation has been implicated in regulation of RNAPII elongation and co-transcriptional processing of nascent RNAs ${ }^{15},{ }^{17}{ }^{22}$. CDK7, the kinase subunit of TFIIH, can phosphorylate both Ser5 and Ser7 and mediates 5' capping of mRNAs ${ }^{21}{ }^{25}$. CDK8, a Mediator-associated kinase, can phosphorylate both Ser 2 and Ser5 in vitro, with a preference for Ser $5^{23}, 25$, but its precise contribution to CTD phosphorylation in vivo remains to be determined. Interestingly, positive functions for Mediator at post-RNAPII recruitment steps have been documented. In mice, knock-out experiments showed that the MED23 subunit is required for expression of the EGR1 gene, yet the effects of MED23 depletion could not be merely explained by impaired RNAPII recruitment ${ }^{26}$. Within the $\mathrm{p} 53$ network, Mediator is strongly recruited during activation of genes carrying paused RNAPII, such as p21 and MDM2, suggesting that it acts at postRNAPII recruitment steps at these loci ${ }^{27}$. The mechanisms by which Mediator may facilitate gene activation after transcription initiation remain to be elucidated.

Multiple variants of the human Mediator complex have been isolated that can be categorized as either 'core' Mediator or CDK8-Mediator based on the absence or presence of the CDK8submodule ${ }^{1}{ }^{28}$. The CDK8-submodule is composed of CDK8, cyclin C, MED12 and MED13 and has been characterized mostly as a transcriptional repressor. For example, in vitro assays show that addition of the CDK8-module shuts down transcription driven by several transactivators ${ }^{29}$. This repression is independent of CDK8 kinase activity and it is likely due to disruption of Mediator-RNAPII interactions ${ }^{29},{ }^{30}$. In vivo, activation of RARa and C/EBP- $\beta$ target genes correlates with a switch from CDK8-Mediator to core Mediator ${ }^{31}, 32$. Additionally, the MED12 subunit mediates the recruitment of repressive histone methyl-transferases in vivo ${ }^{33}$. However, several reports have shown that CDK8 can also function as a positive regulator of gene activity. In yeast, chemical genetics experiments demonstrated that SRB10, the CDK8 ortholog, collaborates with KIN28, the CDK7 ortholog, to promote RNAPII transcription both in vitro and in vivo ${ }^{34}$. In human cells, CDK8 is recruited to specific p53 target genes exclusively during conditions of activation, and CDK8 depletion impairs induction of select genes within this network ${ }^{27}$. Recently, CDK8 was shown to positively regulate $\beta$-catenin-dependent transcription in human cells ${ }^{35}$. Furthermore, CDK8 can collaborate with GCN5L to phosphoacetylate histone H3, a mark that correlates with activation ${ }^{36}$.

A recent report provides several lines of evidence that establish CDK8 as a potent oncoprotein in colon cancer: 1) CDK8 amplification and overexpression is commonly observed in colon tumors, 2) CDK8 knock-down decreases proliferation of several colon cancer-derived cell lines, 3) CDK8 overexpression leads to focus-formation, anchorageindependent growth and tumor formation in immunodeficient animals ${ }^{35}$. These results could 
be explained in part by the fact that CDK8 is required for transcriptional activation by the oncogenic transcription factor $\beta$-catenin/TCF ${ }^{35},{ }^{37}$. However, only a fraction of the malignant effects of CDK8 overexpression could be blocked by a dominant negative form of TCF, suggesting that CDK8 participates in other oncogenic pathways ${ }^{35}$. Given the prominent role of growth factor signalling in cancer development, we hypothesized that CDK8 could have functions within the serum response network. Indeed, we found that human CDK8 is a potent positive regulator of immediate early genes (IEGs), which are strongly transcribed within minutes of growth factor signalling. Microarray experiments in human cells demonstrate that CDK8-dependent coactivation, rather than repression, is predominant within this gene network. These effects are direct, as CDK8 is strongly recruited to these genes during activation and CDK8 depletion leads to impaired RNAPII activity at these loci. We found that CDK8 is not required for recruitment of RNAPII to promoters and overall RNAPII promoter escape. Instead, nuclear run-on experiments demonstrate that RNAPII elongation is impaired upon CDK8 knock-down, which correlates with decreased levels of RNAPII CTD phosphorylation at Ser2 and Ser5. CDK8 regulates precise steps during the assembly of a functional elongation complex, as CDK8 depletion impairs the orchestrated recruitment of CDK7, P-TEFb and its associated factor BRD4 ${ }^{13}, 38$. More interestingly, we show that $\mathrm{P}-\mathrm{TEFb}$ interacts with both the free CDK8-submodule and CDK8-Mediator, suggesting that the CDK8-submodule may help load P-TEFb at the promoters of serum response genes. Thus, our results demonstrate a novel role for CDK8 in transcriptional regulation at post-RNAPII recruitment steps.

\section{Results}

\section{CDK8 is a positive regulator of serum response gene expression}

To determine the role of CDK8 in the expression of serum responsive genes, we analyzed mRNA expression using microarray technology in HCT116 colon cancer cells where CDK8 expression was stably knocked-down. shRNA-mediated depletion of CDK8 results in an 8090\% reduction of CDK8 mRNA and total protein levels (Fig. 1a). Though CDK8 is required for proliferation of multiple colon cancer cell lines, CDK8 is not amplified in HCT116 cells and has a more modest effect on their proliferation, which enabled us to perform the experiments described in this report. Expression of CDC2L6 (CDK8-L), a CDK8 paralog also associated with Mediator ${ }^{28}$, was not affected by our shRNA (Supplementary Fig. 1a). We identified 29 transcripts that were upregulated $>2$-fold after 30 minutes of serum stimulation in wild type cells. Roughly $90 \%$ (26/29) of these immediate early genes (IEGs), were expressed at significantly lower levels ( $<2$-fold) in serum-stimulated shCDK8 cells (Fig. 1b). Only 3 serum-responsive genes (THBS1, PLK2 and KLF6) were expressed at higher levels in shCDK8 cells. A number of IEGs, such as members of the AP-1 (Activating Protein 1) and EGR (Early Growth Response) families of transcription factors stood out (FOS, JUN, FOSB, JUNB and EGR1, 2, 3). Interestingly, these are potent oncogenes acting downstream of MAPK-dependent signalling that are activated by the ubiquitous serum response factor (SRF) and Ets-like transcription factors, such as ELK1 ${ }^{39}$. Validation of microarray results by Q-RT-PCR showed that CDK8 depletion leads to decreased expression of all genes analyzed over multiple time points (Fig. 1c and Supplementary Fig. 1b). Of note, the effect of CDK8 knock-down is more drastic during the early time points, 
when increased gene expression is driven by transcriptional activation and less influenced by subsequent transcriptional shut-down and mRNA degradation. The effects of CDK8 knock-down on IEG expression are not due to off-target effects of a particular shRNA sequence, because reduced expression of IEGs is also observed when targeting a different sequence within the CDK8 mRNA (Supplementary Fig. 1c).

\section{CDK8 is required for RNAPII CTD phosphorylation, not RNAPII recruitment}

To test whether the impact of CDK8 on IEG mRNA expression is due to a direct role of CDK8 in transcriptional activation at these loci we performed chromatin immunoprecipitation assays (ChIP) for CDK8, total RNAPII and two phosphorylated isoforms of RNAPII. ChIP analysis of CDK8 showed that prior to stimulation there is little to no CDK8 at the promoters of FOS and EGR1, 2 and 3. However, serum treatment of wild type cells produces a strong increase in CDK8 recruitment (7-14 fold) (average data for FOS and EGR1-3 is shown in Fig. 2, statistical analysis is shown in Supplementary Fig. 2). As expected, CDK8 occupancy decreases 4-9 fold in shCDK8 cells across the loci studied. ChIP analysis indicates that CDK8 is not required for the recruitment of RNAPII to all four IEGs, but does have a positive affect on CTD phosphorylation (Fig. 2). RNAPII is preloaded at the FOS proximal promoter with only a small increase in occupancy after serum treatment. However, a clear increase in RNAPII is observed at the FOS and EGR1 intragenic regions during activation, indicative of enhanced elongation. Interestingly, CDK8 knock-down produces no substantial effects on total RNAPII occupancy at any of the locations tested, before or after activation. This is more evident at the non-preloaded EGR2 and EGR3 genes, which show a sharp increase in RNAPII occupancy at their proximal promoters upon serum treatment ( 4-7 fold) that is refractory to CDK8 depletion (Fig. 2). RNAPII CTD phosphorylation at Ser5 and Ser2 is linked to promoter clearance and elongation ${ }^{14},{ }^{15}$. Accordingly, both phosphorylation marks increase sharply upon activation at IEGs in wild type cells. Typically, Ser5 phosphorylation peaks near the transcription start site, whereas Ser2 phosphorylation accumulates at the $3^{\prime}$ end of genes ${ }^{18}$. Depletion of CDK8 clearly reduces the level of Ser5 phosphorylation (S5P) at all three EGR genes, however the effects on FOS are less pronounced. More strikingly, CDK8 knock-down strongly reduces the level of phospho-Ser2 (S2P) at all four genes (Fig. 2). Importantly, CDK8 knock-down does not affect cellular levels of either mark as measured by western blot (Supplementary Fig. 3a), indicating that CDK8 effects on CTD phosphorylation are gene-specific rather than global. Of note, CDK8 knock-down does not affect mRNA expression or RNAPII activity at the housekeeping gene GAPDH (Supplementary Fig. 3b and 3c). In summary, our results indicate that CDK8 is playing a role in transcriptional activation events subsequent to recruitment of RNAPII at serum-responsive loci.

\section{CDK8 affects the rate of transcriptional elongation at IEGs}

Intriguingly, our ChIP analysis did not detect significant differences in RNAPII occupancy within IEGs intragenic regions, yet clear effects on CTD phosphorylation and mRNA steady-state levels were observed. CTD phosphorylation is known to affect both RNAPII elongation and cotranscriptional RNA processing. To investigate the impact of CDK8 depletion on these events, we first measured the levels of unprocessed primary transcripts using PCR primers amplifying intronic regions. Clearly, CDK8 depletion causes a sharp 
decrease in the levels of FOS and the EGR pre-mRNAs produced upon serum stimulation (Fig. 3a), suggesting that the CDK8-dependent effects on CTD phosphorylation result in a defect in the rate of transcription rather than co-transcriptional RNA processing. To test this, we performed nuclear run-on assays in conditions that prevent re-initiation. Serum starved cells were treated with serum for 10 minutes before active elongation complexes were stalled and nuclei isolated. Transcription was then allowed to progress for either 5 or 60 minutes before nuclei were lysed. Analysis of primary transcripts generated during the runon shows that at the 5 minute time point RNA levels of FOS, EGR1-3 are 2-20 fold higher in control cells as compared to shCDK8 cells; however, by the 60 minute time point the RNA levels are either equivalent or much more similar (Fig. 3b). This provides strong evidence that CDK8 is positively affecting the actual rate of transcription. Although RNAPII still elongates in CDK8-depleted cells, it does so at a slower pace.

\section{CDK8 is required for assembly of elongation complexes at serum response genes}

In agreement with the fact that CDK8 plays no role in RNAPII recruitment, we found that CDK8 depletion did not affect recruitment or activation of the key transactivators acting at the FOS promoter. The SRF-ELK1 complex is constitutively bound to the chromatin of its target genes and is activated by MAPK-dependent phosphorylation ${ }^{39}$. Phosphorylation of the C-terminal domain of ELK1 has been shown to be important for the allosteric stimulation of histone acetyl-transferase (HAT) activities ${ }^{40}$. CDK8 depletion did not affect the levels of chromatin bound SRF or ELK1 (condensed data is shown Fig. 4, statistical analysis is shown in Supplementary Fig. 4). Likewise, we did not observe differences in ELK1 phosphorylation or overall histone acetylation (AcH4) (Fig. 4a). Expectedly, CDK8 did not affect the chromatin-bound levels of Pre-Initiation Complex (PIC) components involved in RNAPII recruitment, such as TBP or TFIIB (Fig. 4a).

It has been demonstrated that phosphorylation of ELK1 triggers association with the MED23 subunit of Mediator and that MED23 is required for activation of certain ELK1target genes ${ }^{41}$. Our ChIP analysis confirms that MED23 is recruited to FOS and EGR1 upon serum stimulation (Fig. 4b). Interestingly, the levels of chromatin-bound MED23 are decreased by roughly 50\% in CDK8 knock-down cells, suggesting that CDK8 helps stabilize Mediator association at these loci. This notion is supported by the fact that two other Mediator subunits, MED1 and MED12, also show decreased association to the FOS and EGR1 promoters upon CDK8 depletion (Fig. 4b). Whereas CDK8 knock-down may have a modest effect on MED12 stability, it does not decrease the cellular pools of MED23 and MED1 (Supplementary Fig. 3a).

Given the clear effects of CDK8 knock-down on RNAPII CTD phosphorylation and elongation rates at IEGs, we next focused on complexes containing CTD-kinases. We found that recruitment of both CDK7 and CDK9 to the FOS and EGR1 loci is impaired upon CDK8 knock-down (average data for FOS and EGR1 is shown in Fig. 5, statistical analysis is shown in Supplementary Fig. 5). In contrast to CDK7 and CDK9, the recruitment of other factors regulating RNAPII pausing and elongation, such as NELF, DSIF and FACT, is not affected by CDK8 knock-down. NELF and DSIF (a dimer of SPT4 and SPT5) are key regulators of RNAPII pausing at promoters ${ }^{42},{ }^{43}$. Upon promoter escape, SPT5 associates 
with RNAPII and functions as a positive elongation factor ${ }^{43},{ }^{44}$. FACT (a dimer of SSRP1 and SPT16) is another positive regulator of elongation required for transcription-coupled nucleosome remodelling ${ }^{45}$. Our ChIP analysis demonstrates that NELF-A and SPT5 occupy the FOS promoter carrying preloaded RNAPII. Upon activation, NELF-A remains at the transcription start site whereas SPT5 associates with the intragenic region. In contrast, SPT16 is recruited strongly to the gene body upon serum treatment (Fig. 5). Occupancy profiles for all three factors are unaffected by CDK8 depletion during both basal and stimulated conditions. These results indicate that CDK8 orchestrates key events in the formation of a functional elongation complex, namely, recruitment of CDK7 and CDK9 and subsequent RNAPII CTD phosphorylation.

\section{CDK8 depletion impairs BRD4 recruitment without effects on histone acetylation}

The bromodomain protein BRD4 has been recently implicated in recruitment of P-TEFb to several human genes ${ }^{38},{ }^{46}$. BRD4 interacts with both acetylated histones and P-TEFb, thus linking chromatin modifications to elongation control. Importantly, several reports described an interaction between BRD4 and Mediator, yet the relevance of this interaction has not been defined ${ }^{38},{ }^{47}{ }^{49}$. Our ChIP analyses demonstrate that BRD4 is strongly recruited to FOS and EGR1 upon serum stimulation with a profile similar to that of CDK9 and Mediator. Interestingly, BRD4 recruitment is significantly decreased upon CDK8 knockdown. Strikingly, our detailed analysis of histone acetylation at FOS and EGR1 indicates that BRD4 recruitment is most likely due to its interaction with Mediator rather than its association with the acetyl-lysines previously described to be recognized by its double bromodomain. Hargreaves et al concluded that BRD4 detects histone H4 acetylated at lysines 5, 8 and $12^{46}$. In contrast, Zippo et al concluded that BRD4 binds instead histone H4 acetylated at lysine 16 and histone $\mathrm{H} 3$ acetylated in lysine $9^{38}$. Our ChIP analyses using antibodies against tetra-acetyl H4 (lysines 5, 8, 12, 16), acetyl-H4-K8 (H4 K8Ac), acetylH4-K12 (H4 K12Ac), acetyl-H4-K16 (H4 K16Ac) and acetyl-H3-K9 (H3 K9Ac) demonstrate that none of these marks is affected by CDK8 knock-down and that the occupancy profiles of these marks do not resemble that of BRD4 (Figs. 4a, 5a and Supplementary Figs. 5 and 6).

\section{CDK9 inhibition reproduces the effects of CDK8 knock-down on RNAPII activity}

Our results indicate that many of the effects of CDK8 knock-down on RNAPII activity at post-recruitment steps could be explained by decreased $\mathrm{P}-\mathrm{TEFb}$ recruitment. To test for the impact of CDK9 activity on IEG activation, we employed the pharmacological inhibitor flavopiridol ${ }^{10}$. Pre-treatment of cells with flavopiridol $(150 \mathrm{nM})$ prevents serum-induced mRNA accumulation of FOS, EGR1-3 (Fig. 6a). ChIP analysis demonstrates that CDK9 inhibition does not decrease the levels of promoter-bound RNAPII at IEGs carrying preloaded RNAPII (e.g. EGR1) or those displaying induced RNAPII recruitment (e.g. EGR2) (Fig. 6b). Clearly, inhibition of CDK9 by flavopiridol has a more pronounced effect on RNAPII occupancy at intragenic regions than CDK8 depletion, which could be explained by the fact that some amount of P-TEFb is still recruited to these loci in CDK8-knock-down cells. However, a small but significant amount of intragenic RNAPII is still detected in flavopiridol-treated cells. These RNAPII complexes presumably elongate at such low rates that prevent significant mRNA accumulation. Expectedly, flavopiridol treatment completely 
blocks RNAPII CTD phosphorylation in Ser2. Flavopiridol also blocks Ser5 phosphorylation in intragenic regions, but has no effect on promoter-proximal Ser5 phosphorylation. Overall, these results suggest that decreased CDK9 activity at IEGs upon CDK8 depletion could explain the post-recruitment defects observed in CDK8 knock-down cells.

\section{CDK8-Mediator interacts with P-TEFb}

A role for Mediator in recruitment of TFIIH has already been described in other systems ${ }^{50}$, which could explain the reduced association of CDK7 to IEG promoters upon CDK8 depletion. However, it is unclear how CDK8-Mediator may impact P-TEFb recruitment. At least two variants of the human Mediator complex exist within the cell nucleus ${ }^{1}$. CDK8Mediator complexes contain the CDK8-submodule and are devoid of the MED26 subunit, which is unique to 'core' Mediator, whereas the MED1 subunit is found in both. We have previously reported that a large fraction of the CDK8-submodule exists separate from core Mediator $^{51}$. Serendipitously, research efforts to identify factors interacting with Mediator showed that $\mathrm{P}-\mathrm{TEFb}$ associates specifically with CDK8-Mediator. In these experiments, antibodies against CDK8 or MED1 were used to immunoprecipitate Mediator from nuclear extracts. After a series of high-salt washes, bound proteins were eluted and identified using MudPIT methodology. Interestingly, multiple peptides for CDK9 and cyclin T were detected in the CDK8 IP, but not in the MED1 IP (Ebmeier and Taatjes, unpublished results). These results were confirmed by quantitative western blot experiments (Fig. 7b). Expectedly, subunits of the CDK-submodule (MED12, MED13 and Cyclin C) are enriched in the CDK8 IP, whereas MED26 was not detected in these CDK8 IP samples. In agreement with several independent reports ${ }^{38},{ }^{47}{ }^{49}$, we also observed an association of BRD4 with Mediator. Interestingly, and in agreement with $\mathrm{Wu}$ and Chiang, we find that BRD4 associates with Mediator regardless of the presence of the CDK8-submodule ${ }^{48}$. Importantly, quantitative western blotting clearly shows enrichment of the P-TEFb subunits CDK9 and Cyclin T1 in the CDK8-Mediator sample (Fig. 7b).

In order to investigate if $\mathrm{P}-\mathrm{TEFb}$ interacts with CDK8-Mediator and/or the free CDK8submodule, we tested for the presence of CDK9 in fractions enriched for these complexes via biochemical fractionation. Using purification methods previously described ${ }^{51}$, we found that CDK9 copurifies with both CDK8-Mediator and the free CDK8-module (Fig. 7c). These results suggest that the CDK8-module may facilitate association of P-TEFb with the rest of Mediator to regulate CTD phosphorylation and transcriptional elongation at specific gene loci such as the IEGs studied here.

\section{Discussion}

The CDK8-submodule of Mediator is a pleiotropic regulator of gene expression. On one hand, this protein complex has been characterized as a transcriptional repressor that inhibits RNAPII by multiple mechanisms, including: 1) kinase-independent allosteric regulation of Mediator-RNAPII interactions ${ }^{29}$; 2) kinase-dependent inactivation of TFIIH via phosphorylation of its cyclin $\mathrm{H}$ subunit ${ }^{52}$; 3 ) gene silencing via recruitment of histone methyl-transferases ${ }^{33}$. In contrast, several reports indicate that CDK8 is a positive regulator 
of gene activity in other scenarios. In yeast, SRB10 promotes RNAPII activity in vitro and in vivo ${ }^{34}$. In human cells, CDK8 depletion impairs activation of genes responsive to p53 and $\beta$-catenin ${ }^{27},{ }^{35}$. Additionally, CDK8-Mediator complexes can perform histone modifications associated with gene activation ${ }^{36}$. In this report, we provide evidence that CDK8 is a direct positive regulator of transcription within the serum response network. We found that CDK8 is dispensable for RNAPII recruitment to promoters carrying pre-loaded RNAPII (FOS, EGR1) as well as promoters displaying inducible RNAPII recruitment (EGR2, EGR3). Furthermore, CDK8 depletion did not produce a drastic impairment in RNAPII escape from promoter regions, as significant amounts of RNAPII were detected throughout the intragenic regions of these genes upon activation. Instead, decreased IEG expression in CDK8 knockdown cells is explained by the appearance of slower elongation complexes containing hypophosphorylated RNAPII. The findings in this report represent a marked advance in our understanding of how CDK8 promotes gene activity, but also prompt more general questions about the role of CTD phosphorylation, CTD kinases and Mediator in mammalian gene expression control.

Here, we establish a strong correlation between CDK8 status, CTD phosphorylation and RNAPII elongation rates. Although some effect of CDK8 depletion on IEG RNA processing can not be fully discarded, our results indicate that most of the negative effects of CDK8 knock-down on IEG expression are explained by decreased elongation rates. In nuclear runon experiments, CDK8 depletion decreases elongation rates by $\sim 2-3$-fold at the FOS locus and $>10$-fold at the EGR loci. Importantly, these differences are attenuated when additional time is allowed for elongation complexes derived from CDK8-depleted cells to proceed, with the FOS locus showing equivalent RNA synthesis and the EGR loci reaching up to 30$60 \%$ of wild type levels by 60 minutes. Interestingly, the impact of CDK8 on CTD phosphorylation is more pronounced at the EGR loci, further supporting a link between CTD phosphorylation and elongation rates. The notion that decreased transcription, rather than impaired processing, causes lower IEG mRNA expression in CDK8-depleted cells is reinforced by the fact that these cells accumulate lower levels of pre-mRNAs.

Despite the fact that CDK8 is a CTD-kinase and that its depletion leads to hypophosphorylated CTD and slower elongation complexes, a simple interpretation of these results is complicated by the fact that recruitment of two other CTD-kinases, CDK7 and CDK9, is impaired by CDK8 depletion. Until recently, the prevalent view on CTD phosphorylation maintained that CDK7 was the major Ser5-kinase and CDK9 the predominant Ser2-kinase. This view originated with the early characterization of CDK7 and the CDK9-like kinases in yeast ${ }^{16},{ }^{53}$. However, recent chemical genetic experiments in yeast and human cells showed that inhibition of CDK7 kinase activity does not result in global defects in Ser5 phosphorylation ${ }^{24}{ }^{54},{ }_{5}$; but CDK7 may instead be the major kinase for Ser7, an alternate phospho-acceptor site within the CTD ${ }^{24},{ }^{56}$. Interestingly, CDK9 may be a relevant Ser5-kinase in certain contexts ${ }^{57}, 58$. CDK8 is known to phosphorylate both Ser2 and Ser5 in vitro ${ }^{23}$, but its in vivo contributions remain to be elucidated. Interplay between the CTD kinases is supported by the fact that Ser5 phosphorylation by CDK7 'primes' the CTD for subsequent Ser2 phosphorylation by CDK9 ${ }^{59}$. Yeast Mediator was shown to be required for TFIIH recruitment to specific promoters and our data suggest that a similar interaction between CDK8-Mediator and TFIIH may also take place at IEGs ${ }^{50}$. 
Additionally, our data indicate that P-TEFb association with the RNAPII machinery is also facilitated by CDK8-Mediator and we provide evidence of a physical interaction between the two complexes.

Though much is known regarding the critical role of $\mathrm{P}-\mathrm{TEFb}$ in elongation control, little is known about the mechanisms by which this complex is recruited to allow for signalling- and gene-specific regulation. Several modes of $\mathrm{P}-\mathrm{TEFb}$ recruitment have been described, including direct interactions with DNA-binding proteins or with the bromodomain protein BRD4 ${ }^{13}, 38,48,49,60$. Interestingly, BRD4 has been shown repeatedly to interact with Mediator and our results confirm this interaction. Furthermore, our results agree with those of $\mathrm{Wu}$ and Chiang who found that BRD4 associates with both CDK8-Mediator and core Mediator $^{48}$. In contrast, we find that $\mathrm{P}-\mathrm{TEFb}$ associates preferentially with CDK8-Mediator and the free CDK8-submodule. These observations suggest that BRD4 and P-TEFb interact with Mediator by different means.

Early evidence that Mediator regulates RNAPII activity at post-recruitment steps was demonstrated by Wang et $\mathrm{al}^{26}$. Using MED23 null mice, they found that abolishing Mediator recruitment to the EGR1 locus drastically impairs transcriptional activation without affecting binding of TFIIA, TFIID, histone acetylation, methylation or association of the chromatin remodelling factor BRG1. Importantly, the defects in EGR1 transcription observed in MED23-/- cells could not be merely explained by reduced RNAPII association, as a significant fraction of RNAPII remained associated with the promoter in mutant cells. Thus, they concluded that Mediator affected both recruitment and post-recruitment steps. Given that RNAPII occupancy remains unaffected in CDK8 knock-down cells, we have been able to more clearly define the contribution of Mediator to post-recruitment steps. A significant fraction of MED23 remains associated with the promoter upon CDK8 depletion, which may explain why we do not observe any recruitment defects. Instead, most effects of CDK8 knock-down on IEG expression can be explained by impaired P-TEFb recruitment. This notion is supported by the fact that CDK9 inhibition by flavopiridol resembles (albeit with more penetrance) the effects of CDK8 knock-down. Given past results that demonstrate mutually exclusive association of CDK8-submodule and RNAPII with Mediator ${ }^{28}$, ${ }^{29}$, our data further suggest dynamic association of the CDK8-submodule at the promoter of active serum response genes. Taken together, these results invoke a mechanism involving CDK8submodule exchange at the promoter to regulate RNAPII and P-TEFb association within the transcriptional apparatus at post-recruitment steps.

CDK8 is a potent oncogene in colon cancer. CDK8 overexpression promotes cell proliferation, anchorage-independent growth, and tumor growth in xenografts 35 . Part of these effects can be attributed to the fact that $\mathrm{CDK} 8$ promotes transcription mediated by the oncogenic transcription factor $\beta$-catenin/TCF ${ }^{35}$. Here, we report that CDK8 is a potent positive regulator of the serum response network, which has been implicated in multiple tumorigenic phenotypes. The precise contributions of the $\beta$-catenin and serum response networks to the overall oncogenic effects of CDK8 overexpression await further experimentation. Whereas the transformation-inducing effects of $\beta$-catenin can be effectively blocked by overexpression of a dominant negative version of its binding partner $\mathrm{TCF}^{35}$, the equivalent task for the serum response network is not straightforward. Upon 
serum stimulation, ERKs translocate to the nucleus and activate a myriad of transcription factors, including multiple members of the Ets family, MYC and C/EBP- $\beta^{39}$. Possible functional redundancy within the Ets family will impose a combinatorial genetic approach to determine their precise interaction with CDK8 during tumor development. Nonetheless, the fact that many MAPK-regulated transcription factors and their target genes have well demonstrated roles in cancer development will make this a worthy task that would lead to a detailed understanding of the oncogenic effects of CDK8 overexpression.

\section{Methods}

Cell Culture

HCT116 cells were maintained in McCoys 5A media supplemented with $10 \%$ (v/v) fetal bovine serum (Hyclone) and antibiotic/antimycotic mix (Gibco-Invitrogen). HEK293FT cells were grown in DMEM media supplemented with $10 \%$ (v/v) fetal bovine serum and antibiotic/antimicotic mix (Gibco-Invitrogen). For western blot, all Q-RT-PCR assays and ChIP, cell cultures at 50-70\% confluency were washed twice with PBS, starved in serum free media for greater than 40 hours and then treated with serum-containing media for the indicated times. For flavopiridol treatments $(150 \mathrm{nM})$, the drug was added $1 \mathrm{~h}$ prior to serum stimulation.

\section{shRNA- and siRNA- mediated knock-down}

Stable clones constitutively expressing a short-hairpin RNA targeting CDK8 (shCDK8) were generated using the lentiviral-based shRNA delivery vector pLL3.7. The CDK8 mRNA sequence targeted by the shCDK8 is 5'-GGATGATAAAGACTATGCT-3'. Viral particles were produced in HEK293FT cells and used to transduce HCT116 cells, which were subsequently cloned in the presence of $1 \mu \mathrm{g} \mathrm{ml}^{-1} \mathrm{G} 418$ (Sigma). For transient CDK8 knock-down, cells were starved two hours prior to transfection, with control siGlo siRNA (Dharmacon) or CDK8 specific siRNA (Invitrogen), using Lipofectamine (Invitrogen) per the manufacturers protocols. 40 hours post-transfection, cells were treated with complete media for the indicated times before harvesting for Q-RT-PCR analysis. The sequence targeted by the CDK8 siRNA is 5'-CCCAATAAAGCGAATTACCTCAGAA-3'.

\section{Western Blots}

These assays were performed as described in ${ }^{58}$. For western blots, $10 \mu \mathrm{g}$ of total protein extract were loaded onto SDS-PAGE gels, transferred to PVDF membranes and blotted with the appropriate antibodies. Antibody information can be found in Supplementary Table 1.

\section{Q-RT-PCR}

Total RNA was isolated using the RNeasy Extraction Kit (Qiagen) and cDNA was generated using the iScript cDNA Synthesis Kit (BioRad). Relative mRNA levels, normalized to $18 \mathrm{~S}$ rRNA, were determined using SYBR Green I chemistry in an ABI Prism 7900 HD RT-PCR machine (Applied Biosystems). See Supplementary Table 2 for primer sequences. 


\section{Microarray analysis}

Serum starved HCT116 cells were left untreated or treated with 10\% (v/v) FBS McCoy's 5A for 30 minutes. RNA was isolated as described above for Q-RT-PCR. Duplicate biological samples were treated according to manufacturers protocols and hybridized to the Human Gene 1.0 ST Arrays (Affymetrix). The raw data from the *.CEL files was imported into SpotFire (TIBCO), normalized using Robust Multi-chip Average (RMA) and analyzed using the Spotfire Decision Site.

\section{ChIP Assays}

ChIP was performed as previously described ${ }^{58}$. Briefly, serum starved HCT116 cells were either treated or not with complete media for 15 minutes, fixed with $1 \%(\mathrm{v} / \mathrm{v})$ formaldehyde, harvested for whole cell lysate preparation and ChIP enriched DNA was analyzed by quantitative-PCR as described ${ }^{58}$. Antibody information can be found in Supplementary Table 1 and oligonucleotide sequences in Supplementary Tables 3-7.

\section{Nuclear Run-On}

Serum starved HCT116 cells were either untreated or treated with 10\% (v/v) FBS McCoy's $5 \mathrm{~A}$ for ten minutes prior to isolation of nuclei. Nuclei from $10^{7}$ cells were isolated in Run-on Lysis Buffer (10 mM Tris- $\mathrm{HCl} \mathrm{pH} 7.5,5 \mathrm{mM} \mathrm{MgCl}_{2}, 10 \mathrm{mM} \mathrm{NaCl}$ and $0.5 \%$ (v/v) NP-40). Nuclei were washed once in $1 \mathrm{x}$ Run-on Reaction Buffer without NTPs $(20 \mathrm{mM}$ Tris-HCl pH 7.5, $10 \mathrm{mM} \mathrm{MgCl} 2,150 \mathrm{mM} \mathrm{KCl}, 20 \%$ (v/v) glycerol), then the run-on was performed for the indicated times at $37 \mathrm{C}$ in the presence of Run-on Reaction Buffer with NTPs ( $1 \mathrm{x}$ Run-on Reaction Buffer, 0.06\% (w/v) Sarkosyl, 0.5 mM ATP, CTP and GTP and either 0.2 mM UTP or $0.2 \mathrm{mM}$ biotin-16-UTP). Run-on reactions were stopped by snap freezing in liquid nitrogen, RNA was purified using RNeasy Extraction Kit (Qiagen) and labeled RNA was further purified using streptavidin-coated Dynabeads (Invitrogen). cDNA was generated and quantified using methods described above for Q-RT-PCR.

\section{Co-immunoprecipitations}

Immunoprecipitation of Mediator complexes was performed by using anti-CDK8 (C-19 Santa Cruz Biotechnology) or anti-MED1 (M-255 Santa Cruz Biotechnology) immobilized on $50 \mu \mathrm{l}$ Protein A/G Sepharose (Amersham Biosciences). After rocking approximately 2 hours at $4 \mathrm{C}$, beads were washed three times with 20 column volumes $0.5 \mathrm{M} \mathrm{KCl} \mathrm{HEGN}$ (20mM Hepes, pH7.6; 0.1 mM EDTA; 10\% (v/v) Glycerol; 0.1\% (v/v) NP-40 alternative; 1 $\mathrm{mM}$ DTT; $1 \mathrm{mM}$ benzamidine; $0.25 \mathrm{M}$ PMSF; $2 \mu \mathrm{g} \mathrm{ml}^{-1}$ aprotinin) and twice with 20 column volumes $0.15 \mathrm{M} \mathrm{KCl} \mathrm{HEGN,} 0.02 \%$ (v/v) NP-40 alternative and incubated with 1.5 $\mathrm{mL} \mathrm{HeLa}$ nuclear extract rocking overnight at $4 \mathrm{C}$. Beads were then washed again three times with 20 column volumes $0.5 \mathrm{M} \mathrm{KCl} \mathrm{HEGN} \mathrm{and} \mathrm{twice} \mathrm{with} 20$ column volumes 0.15 $\mathrm{M} \mathrm{KCl} \mathrm{HEGN,} 0.02 \%$ (v/v) NP-40 alternative. Bound material was eluted twice with $50 \mu \mathrm{l}$ $0.1 \mathrm{M}$ Glycine $\mathrm{pH}$ 2.7. Eluted proteins were separated by SDS-PAGE and resolved by silver staining or immunoblotting. Immunoblotting experiments analyzing serial dilutions of IP elutions were probed using antibodies against the indicated proteins. Antibody information is in Supplementary Table 1. 


\section{Biochemical fractionation of CDK8-containing complexes}

The CDK8-submodule and CDK8-Mediator complexes were purified as previously reported $^{51}$.

\section{Supplementary Material}

Refer to Web version on PubMed Central for supplementary material.

\section{Acknowledgments}

This work was supported initially by a grant from the NIH (CA117907) and later by a grant from NSF (MCB-0842974) to the Espinosa lab. JME is a Howard Hughes Medical Institute Early Career Scientist. Work in the Taatjes lab is supported by grants from NIH (PO1 CA112131) and the Ellison Medical Foundation. AJD and CCE were supported in part by NIH training grant T32GM07135. We are thankful to members of the Espinosa lab for contributing ideas and support, especially Marybeth Sechler and Christopher Potts for technical assistance and Nathan Gomes for discussion.

\section{References}

1. Taatjes DJ, Marr MT, Tjian R. Regulatory diversity among metazoan co-activator complexes. Nat Rev Mol Cell Biol. 2004; 5:403-10. [PubMed: 15122353]

2. Kim YJ, Bj Vorklund S, Li Y, Sayre MH, Kornberg RD. A multiprotein mediator of transcriptional activation and its interaction with the C-terminal repeat domain of RNA polymerase II. Cell. 1994; 77:599-608. [PubMed: 8187178]

3. Cantin GT, Stevens JL, Berk AJ. Activation domain-mediator interactions promote transcription preinitiation complex assembly on promoter DNA. Proc Natl Acad Sci U S A. 2003; 100:12003-8. [PubMed: 14506297]

4. Li XY, Virbasius A, Zhu X, Green MR. Enhancement of TBP binding by activators and general transcription factors. Nature. 1999; 399:605-9. [PubMed: 10376604]

5. Kuras L, Struhl K. Binding of TBP to promoters in vivo is stimulated by activators and requires Pol II holoenzyme. Nature. 1999; 399:609-13. [PubMed: 10376605]

6. Guenther MG, Levine SS, Boyer LA, Jaenisch R, Young RA. A chromatin landmark and transcription initiation at most promoters in human cells. Cell. 2007; 130:77-88. [PubMed: 17632057]

7. O'Brien T, Lis JT. RNA polymerase II pauses at the 5' end of the transcriptionally induced Drosophila hsp70 gene. Mol Cell Biol. 1991; 11:5285-90. [PubMed: 1922045]

8. Espinosa JM, Verdun RE, Emerson BM. p53 functions through stress- and promoter-specific recruitment of transcription initiation components before and after DNA damage. Mol Cell. 2003; 12:1015-27. [PubMed: 14580351]

9. Zeitlinger J, et al. RNA polymerase stalling at developmental control genes in the Drosophila melanogaster embryo. Nat Genet. 2007; 39:1512-6. [PubMed: 17994019]

10. Chao SH, Price DH. Flavopiridol inactivates P-TEFb and blocks most RNA polymerase II transcription in vivo. J Biol Chem. 2001; 276:31793-9. [PubMed: 11431468]

11. Eberhardy SR, Farnham PJ. Myc recruits P-TEFb to mediate the final step in the transcriptional activation of the cad promoter. J Biol Chem. 2002; 277:40156-62. [PubMed: 12177005]

12. Zhu Y, et al. Transcription elongation factor $\mathrm{P}-\mathrm{TEFb}$ is required for HIV-1 tat transactivation in vitro. Genes Dev. 1997; 11:2622-32. [PubMed: 9334325]

13. Yang Z, et al. Recruitment of P-TEFb for stimulation of transcriptional elongation by the bromodomain protein Brd4. Mol Cell. 2005; 19:535-45. [PubMed: 16109377]

14. Kim YK, Bourgeois CF, Isel C, Churcher MJ, Karn J. Phosphorylation of the RNA polymerase II carboxyl-terminal domain by CDK9 is directly responsible for human immunodeficiency virus type 1 Tat-activated transcriptional elongation. Mol Cell Biol. 2002; 22:4622-37. [PubMed: 12052871] 
15. Marshall NF, Peng J, Xie Z, Price DH. Control of RNA polymerase II elongation potential by a novel carboxyl-terminal domain kinase. J Biol Chem. 1996; 271:27176-83. [PubMed: 8900211]

16. Cho EJ, Kobor MS, Kim M, Greenblatt J, Buratowski S. Opposing effects of Ctk1 kinase and Fcp1 phosphatase at Ser 2 of the RNA polymerase II C-terminal domain. Genes Dev. 2001; 15:331929. [PubMed: 11751637]

17. Price DH. P-TEFb, a cyclin-dependent kinase controlling elongation by RNA polymerase II. Mol Cell Biol. 2000; 20:2629-34. [PubMed: 10733565]

18. Komarnitsky P, Cho EJ, Buratowski S. Different phosphorylated forms of RNA polymerase II and associated mRNA processing factors during transcription. Genes Dev. 2000; 14:2452-60. [PubMed: 11018013]

19. Kim M, Ahn SH, Krogan NJ, Greenblatt JF, Buratowski S. Transitions in RNA polymerase II elongation complexes at the 3' ends of genes. Embo J. 2004; 23:354-64. [PubMed: 14739930]

20. Ahn SH, Kim M, Buratowski S. Phosphorylation of serine 2 within the RNA polymerase II Cterminal domain couples transcription and 3' end processing. Mol Cell. 2004; 13:67-76. [PubMed: 14731395]

21. McCracken S, et al. 5'-Capping enzymes are targeted to pre-mRNA by binding to the phosphorylated carboxy-terminal domain of RNA polymerase II. Genes Dev. 1997; 11:3306-18. [PubMed: 9407024]

22. Ho CK, Shuman S. Distinct roles for CTD Ser-2 and Ser-5 phosphorylation in the recruitment and allosteric activation of mammalian mRNA capping enzyme. Mol Cell. 1999; 3:405-11. [PubMed: 10198643]

23. Rickert P, Corden JL, Lees E. Cyclin C/CDK8 and cyclin H/CDK7/p36 are biochemically distinct CTD kinases. Oncogene. 1999; 18:1093-102. [PubMed: 10023686]

24. Akhtar MS, et al. TFIIH kinase places bivalent marks on the carboxy-terminal domain of RNA polymerase II. Mol Cell. 2009; 34:387-93. [PubMed: 19450536]

25. Ramanathan Y, et al. Three RNA polymerase II carboxyl-terminal domain kinases display distinct substrate preferences. J Biol Chem. 2001; 276:10913-20. [PubMed: 11278802]

26. Wang G, et al. Mediator requirement for both recruitment and postrecruitment steps in transcription initiation. Mol Cell. 2005; 17:683-94. [PubMed: 15749018]

27. Donner AJ, Szostek S, Hoover JM, Espinosa JM. CDK8 is a stimulus-specific positive coregulator of p53 target genes. Mol Cell. 2007; 27:121-33. [PubMed: 17612495]

28. Sato $\mathrm{S}$, et al. A set of consensus mammalian mediator subunits identified by multidimensional protein identification technology. Mol Cell. 2004; 14:685-91. [PubMed: 15175163]

29. Knuesel MT, Meyer KD, Bernecky C, Taatjes DJ. The human CDK8 subcomplex is a molecular switch that controls Mediator coactivator function. Genes Dev. 2009; 23:439-51. [PubMed: 19240132]

30. Elmlund $\mathrm{H}$, et al. The cyclin-dependent kinase 8 module sterically blocks Mediator interactions with RNA polymerase II. Proc Natl Acad Sci U S A. 2006; 103:15788-93. [PubMed: 17043218]

31. Mo X, Kowenz-Leutz E, Xu H, Leutz A. Ras induces mediator complex exchange on C/EBP beta. Mol Cell. 2004; 13:241-50. [PubMed: 14759369]

32. Pavri R, et al. PARP-1 determines specificity in a retinoid signaling pathway via direct modulation of mediator. Mol Cell. 2005; 18:83-96. [PubMed: 15808511]

33. Ding N, et al. Mediator links epigenetic silencing of neuronal gene expression with $\mathrm{x}$-linked mental retardation. Mol Cell. 2008; 31:347-59. [PubMed: 18691967]

34. Liu Y, et al. Two Cyclin-Dependent Kinases Promote RNA Polymerase II Transcription and Formation of the Scaffold Complex. Molecular and Cellular Biology. 2004; 24:1721-1735. [PubMed: 14749387]

35. Firestein R, et al. CDK8 is a colorectal cancer oncogene that regulates beta-catenin activity. Nature. 2008; 455:547-51. [PubMed: 18794900]

36. Meyer KD, et al. Cooperative activity of cdk8 and GCN5L within Mediator directs tandem phosphoacetylation of histone H3. EMBO J. 2008; 27:1447-57. [PubMed: 18418385]

37. Morris EJ, et al. E2F1 represses beta-catenin transcription and is antagonized by both $\mathrm{pRB}$ and CDK8. Nature. 2008; 455:552-6. [PubMed: 18794899] 
38. Zippo A, et al. Histone crosstalk between H3S10ph and H4K16ac generates a histone code that mediates transcription elongation. Cell. 2009; 138:1122-36. [PubMed: 19766566]

39. Davis RJ. Transcriptional regulation by MAP kinases. Mol Reprod Dev. 1995; 42:459-67. [PubMed: 8607977]

40. Li QJ, et al. MAP kinase phosphorylation-dependent activation of Elk-1 leads to activation of the co-activator p300. EMBO J. 2003; 22:281-91. [PubMed: 12514134]

41. Stevens JL, et al. Transcription control by E1A and MAP kinase pathway via Sur2 mediator subunit. Science. 2002; 296:755-8. [PubMed: 11934987]

42. Yamaguchi Y, et al. NELF, a multisubunit complex containing RD, cooperates with DSIF to repress RNA polymerase II elongation. Cell. 1999; 97:41-51. [PubMed: 10199401]

43. Wada T, et al. DSIF, a novel transcription elongation factor that regulates RNA polymerase II processivity, is composed of human Spt4 and Spt5 homologs. Genes Dev. 1998; 12:343-56. [PubMed: 9450929]

44. Bourgeois CF, Kim YK, Churcher MJ, West MJ, Karn J. Spt5 cooperates with human immunodeficiency virus type 1 Tat by preventing premature RNA release at terminator sequences. Mol Cell Biol. 2002; 22:1079-93. [PubMed: 11809800]

45. Orphanides G, Wu WH, Lane WS, Hampsey M, Reinberg D. The chromatin-specific transcription elongation factor FACT comprises human SPT16 and SSRP1 proteins. Nature. 1999; 400:284-8. [PubMed: 10421373]

46. Hargreaves DC, Horng T, Medzhitov R. Control of inducible gene expression by signal-dependent transcriptional elongation. Cell. 2009; 138:129-45. [PubMed: 19596240]

47. Houzelstein D, et al. Growth and early postimplantation defects in mice deficient for the bromodomain-containing protein Brd4. Mol Cell Biol. 2002; 22:3794-802. [PubMed: 11997514]

48. Wu SY, Chiang CM. The double bromodomain-containing chromatin adaptor Brd4 and transcriptional regulation. J Biol Chem. 2007; 282:13141-5. [PubMed: 17329240]

49. Jang MK, et al. The bromodomain protein Brd4 is a positive regulatory component of P-TEFb and stimulates RNA polymerase II-dependent transcription. Mol Cell. 2005; 19:523-34. [PubMed: 16109376]

50. Esnault C, et al. Mediator-dependent recruitment of TFIIH modules in preinitiation complex. Mol Cell. 2008; 31:337-46. [PubMed: 18691966]

51. Knuesel MT, Meyer KD, Donner AJ, Espinosa JM, Taatjes DJ. The human CDK8 subcomplex is a histone kinase that requires Med12 for activity and can function independently of mediator. Mol Cell Biol. 2009; 29:650-61. [PubMed: 19047373]

52. Akoulitchev S, Chuikov S, Reinberg D. TFIIH is negatively regulated by cdk8-containing mediator complexes. Nature. 2000; 407:102-6. [PubMed: 10993082]

53. Feaver WJ, Svejstrup JQ, Henry NL, Kornberg RD. Relationship of CDK-activating kinase and RNA polymerase II CTD kinase TFIIH/TFIIK. Cell. 1994; 79:1103-9. [PubMed: 8001136]

54. Kanin EI, et al. Chemical inhibition of the TFIIH-associated kinase Cdk7/Kin28 does not impair global mRNA synthesis. Proc Natl Acad Sci U S A. 2007; 104:5812-7. [PubMed: 17392431]

55. Larochelle $\mathrm{S}$, et al. Requirements for $\mathrm{Cdk} 7$ in the assembly of Cdk1/cyclin B and activation of Cdk2 revealed by chemical genetics in human cells. Mol Cell. 2007; 25:839-50. [PubMed: 17386261]

56. Glover-Cutter K, et al. TFIIH-associated Cdk7 kinase functions in phosphorylation of C-terminal domain Ser7 residues, promoter-proximal pausing, and termination by RNA polymerase II. Mol Cell Biol. 2009; 29:5455-64. [PubMed: 19667075]

57. Jones JC, et al. C-terminal repeat domain kinase I phosphorylates Ser2 and Ser5 of RNA polymerase II C-terminal domain repeats. J Biol Chem. 2004; 279:24957-64. [PubMed: 15047695]

58. Gomes NP, et al. Gene-specific requirement for $\mathrm{P}-\mathrm{TEFb}$ activity and RNA polymerase II phosphorylation within the p53 transcriptional program. Genes Dev. 2006; 20:601-12. [PubMed: 16510875]

59. Viladevall L, et al. TFIIH and P-TEFb coordinate transcription with capping enzyme recruitment at specific genes in fission yeast. Mol Cell. 2009; 33:738-51. [PubMed: 19328067] 
60. Barboric M, Nissen RM, Kanazawa S, Jabrane-Ferrat N, Peterlin BM. NF-kappaB binds P-TEFb to stimulate transcriptional elongation by RNA polymerase II. Mol Cell. 2001; 8:327-37. [PubMed: 11545735] 
a

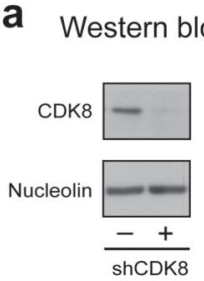

Q-RT-PCR

b

Microarray Heatmaps

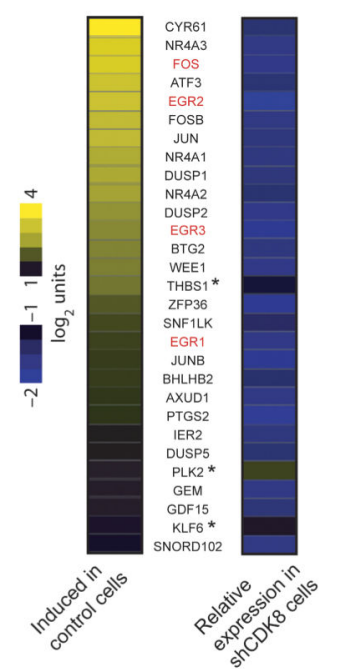

\section{C}
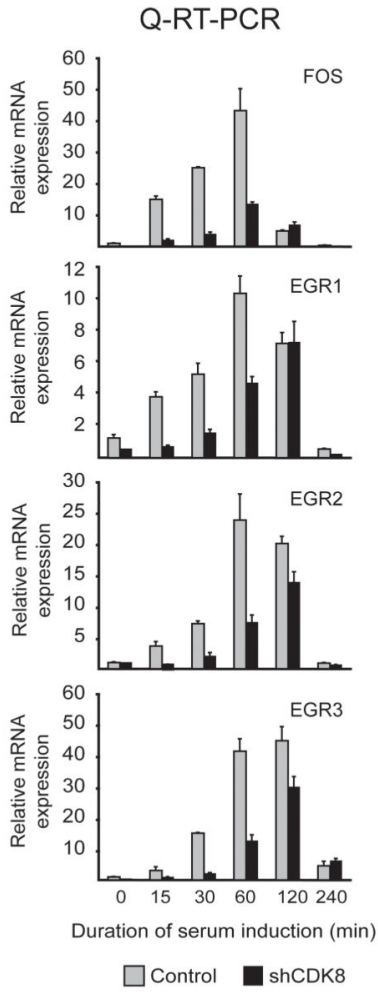

Figure 1. CDK8 is a positive coregulator of serum responsive immediate early genes (a) Western blot and Q-RT-PCR analysis of CDK8 depletion using short-hairpin RNA (shRNA) in HCT116 cells. (b) Microarray. The left panel is a heat map showing the 29 genes induced more than two-fold in control cells. The right panel is a heatmap of the relative level of expression of the same set of genes in CDK8 depleted cells. CDK8dependent activation of the genes highlighted in red is further studied. Asterisks denote the only three genes whose expression increases upon CDK8 depletion. (c) Validation of microarray data. Immediate early gene mRNA expression was measured using quantitativeRT-PCR (Q-RT-PCR). mRNA levels were normalized to 18S rRNA. Data from at least three independent experiments is represented as mean $-/+$ standard error of the mean. 


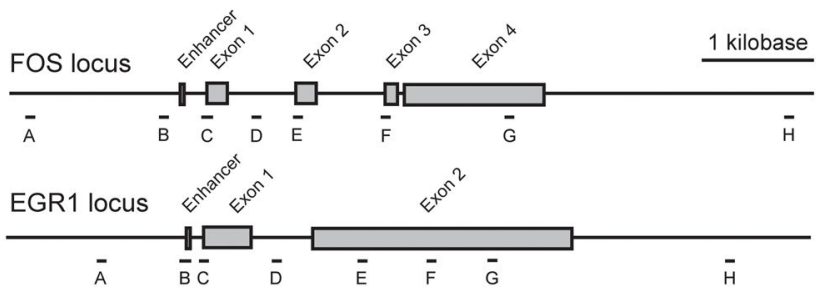

FOS

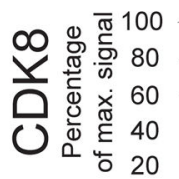

EGR1

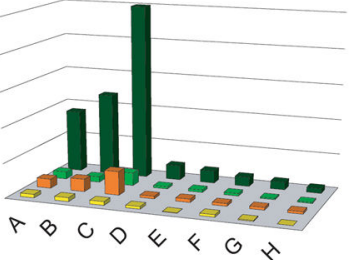

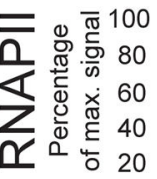
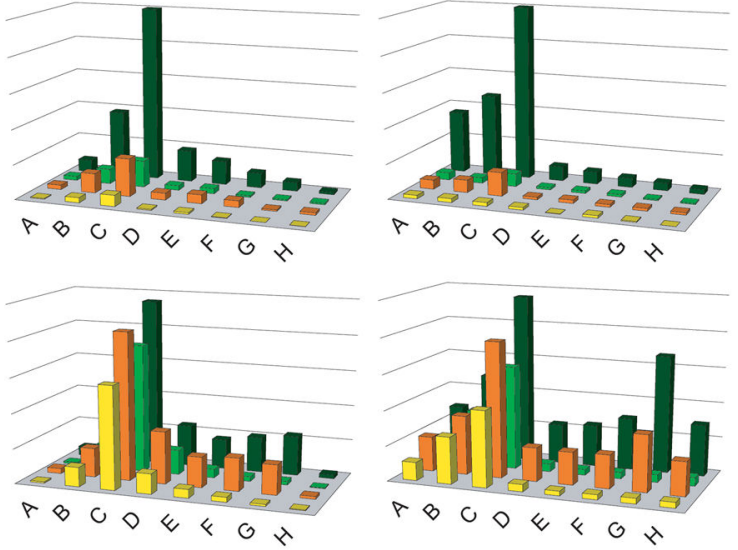

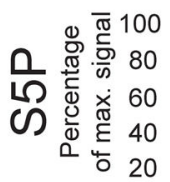
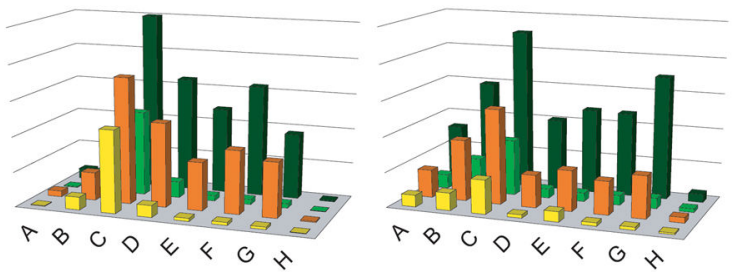

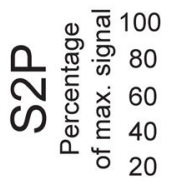

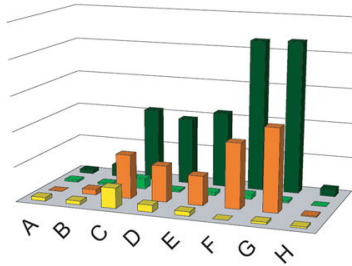

(1) shCDK8

0 min serum

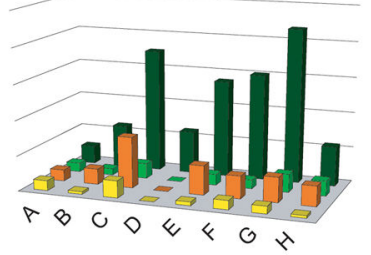

(7) $\mathrm{shCDK8}$

15 min serum

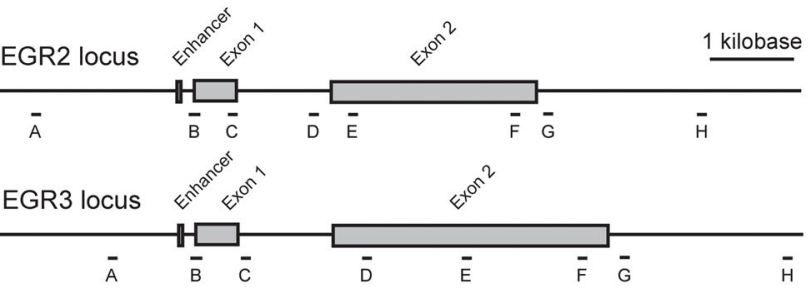

EGR2
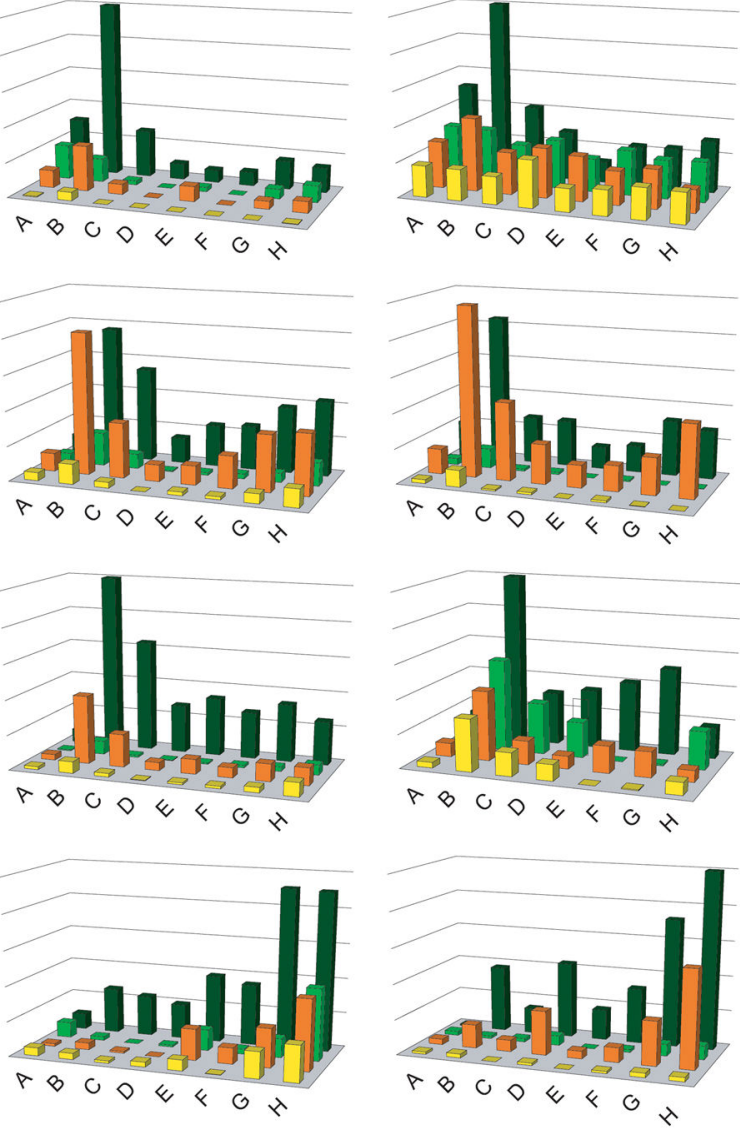

Control

0 min serum
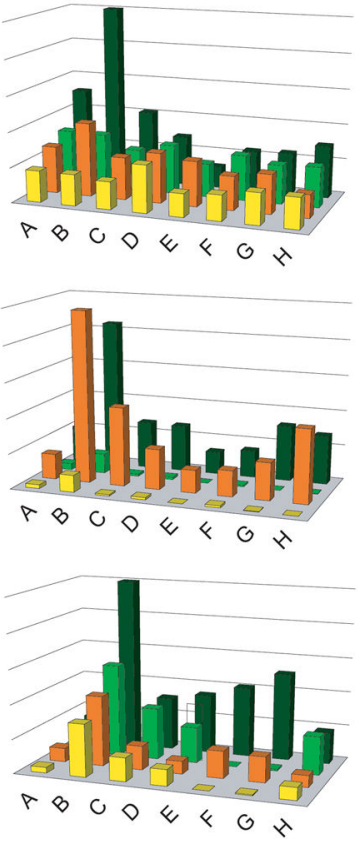

Control

15 min serum

Figure 2. CDK8 positively affects RNAPII CTD phosphorylation at Ser5 and Ser2 without an effect on total RNAPII occupancy at FOS, EGR1, EGR2 and EGR3

The upper panel depicts the basic gene structure (enhancer, exons and introns) of FOS, EGR1, EGR2 and EGR3 and positions of amplicons at each loci used in the ChIP assays. Chromatin immunoprecipitation assay results for cyclin-dependent kinase 8 (CDK8), total RNA polymerase (RNAPII), phospho-Ser5 CTD (S5P) and phospho-Ser2 CTD (S2P) at the FOS, EGR1, EGR2 and EGR3 loci are shown in the lower panel. The mean from at least three independent experiments is represented. 

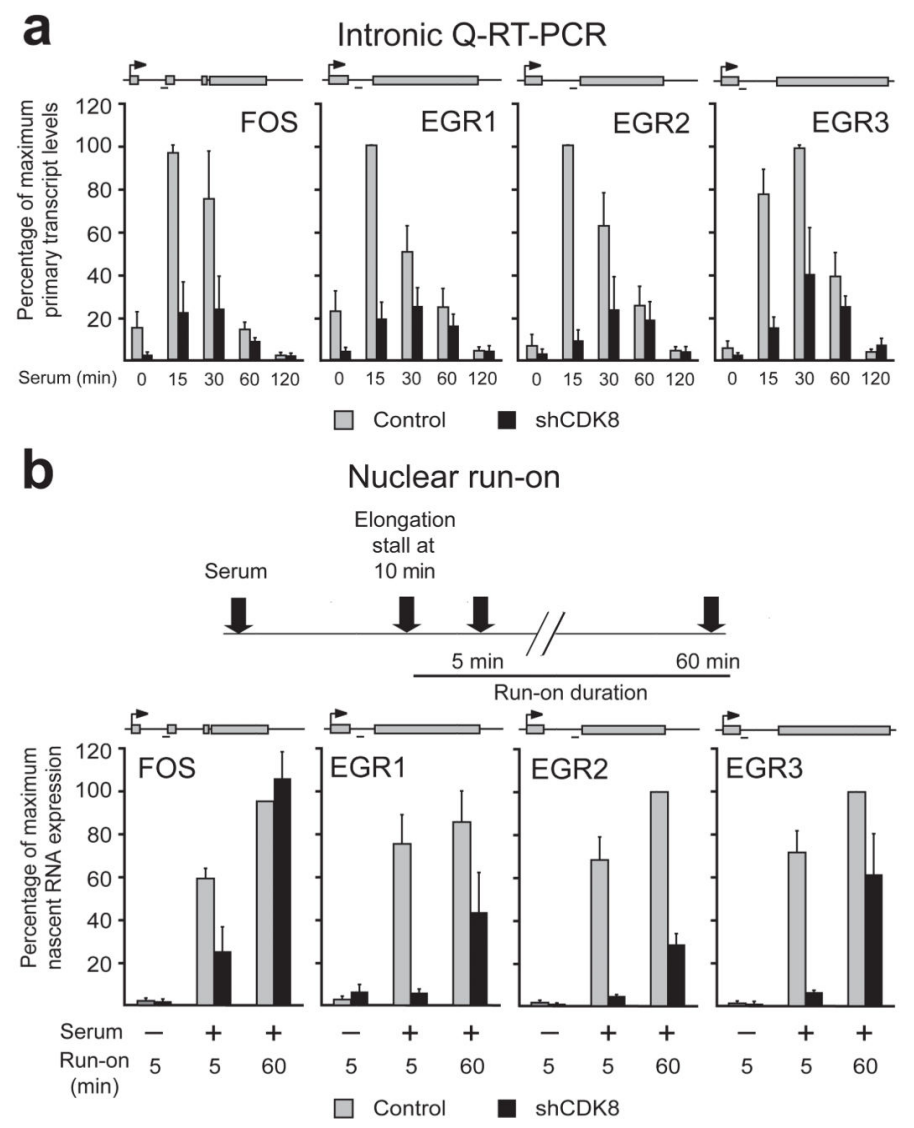

Figure 3. CDK8 promotes RNAPII elongation at IEGs

(a) Q-RT-PCR analysis of IEG primary transcripts. Serum starved cells were treated with serum for the indicated times and nascent message was analyzed by Q-RT-PCR using intronic primers for each gene. (b) Nuclear Run-On analysis. Serum starved cells were treated with serum for 10 minutes, transcription was stalled and then allowed to continue for either 5 or 60 minutes in the presence of biotin-labelled UTP. Labelled RNA was purified and analysed by Q-RT-PCR as in a. Data from at least three independent experiments is represented as mean $-/+$ standard error of the mean. Small schematics of each locus, as depicted in Figures $2 \mathrm{a}$ and $3 \mathrm{a}$, and position of the intronic amplicon used are shown above the expression data for each gene. 
a

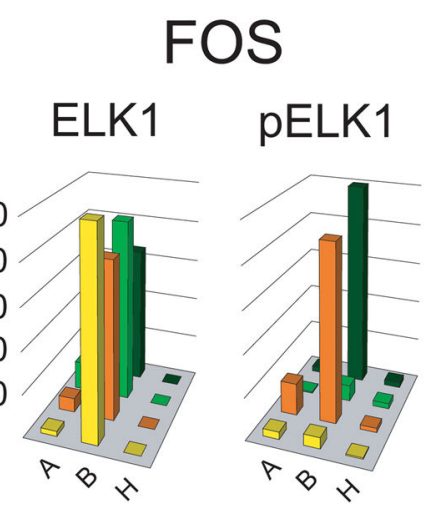

b

FOS

EGR1

MED23

MED23
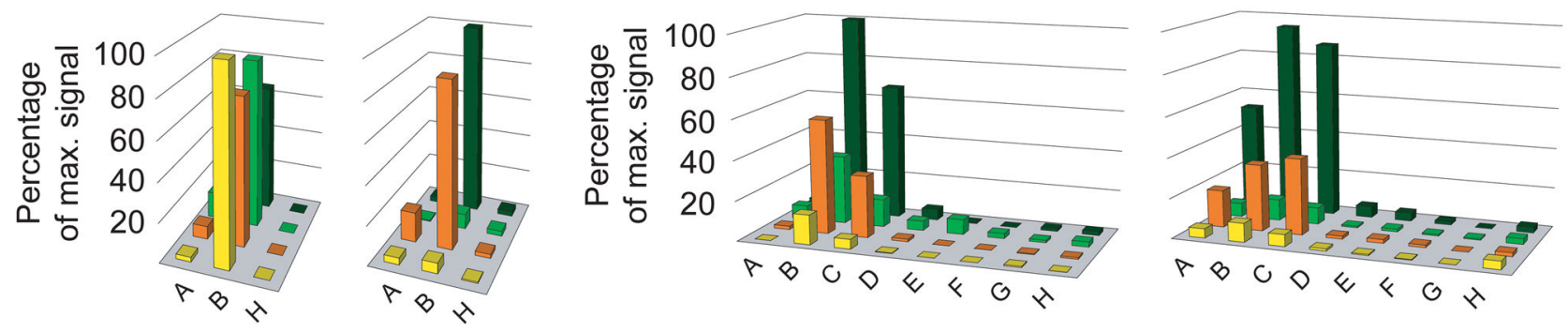

SRF

$\mathrm{AcH} 4$

MED1

MED1
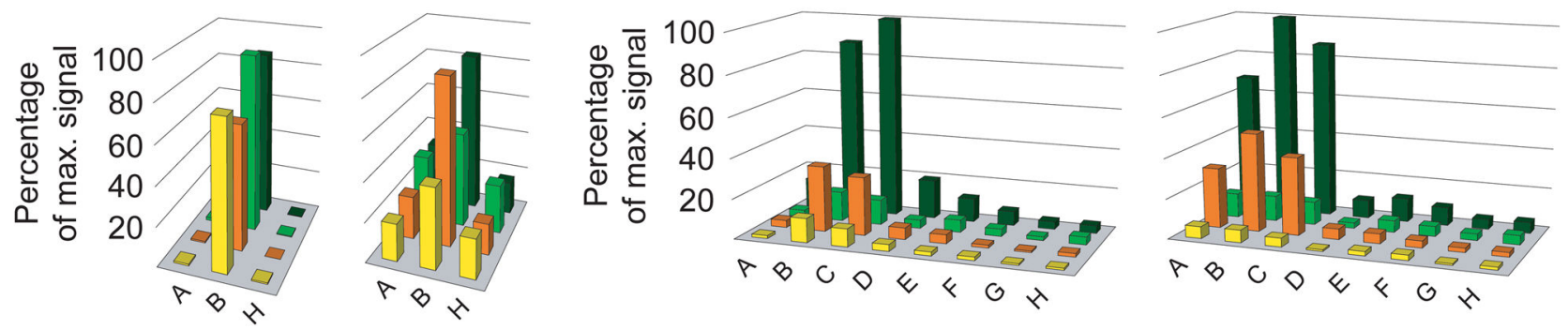

TBP

TFIIB
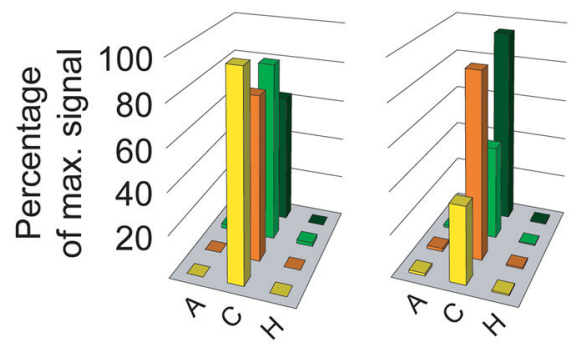

MED12

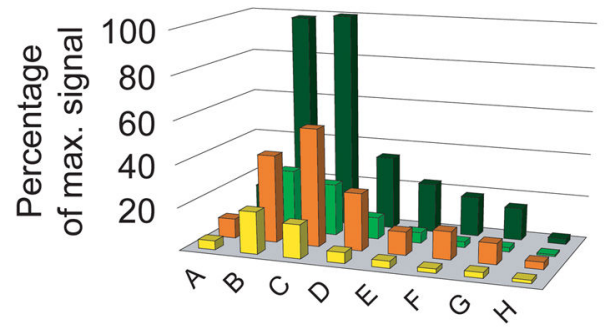

MED12

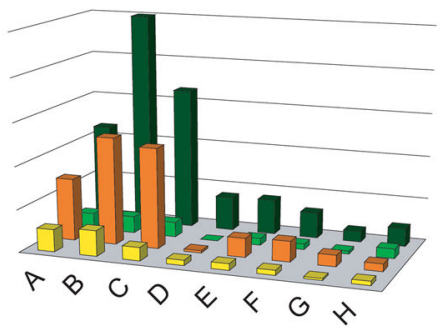

shCDK8 0 min serum

shCDK8 15 min serum

Control 0 min serum

Control 15 min serum

Figure 4. CDK8 affects serum-dependent recruitment of Mediator but not the levels of ELK1 and SRF, ELK1 phosphorylation, tetra-acetyl histone H4 (AcH4), TBP, and TFIIB

ChIP assays with the indicated antibodies and FOS and/or EGR1 directed primers were performed as described in Figure 2. Average data from at least three independent experiments is represented. 
a

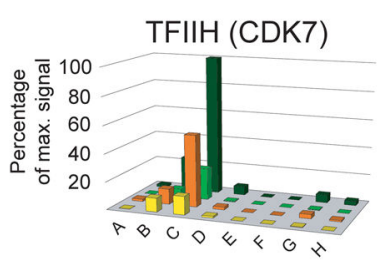

DSIF (SPT5)

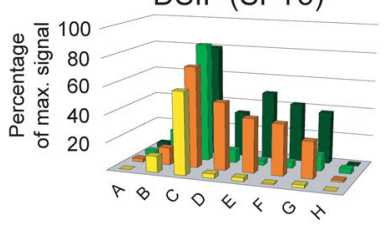

H4 K8Ac

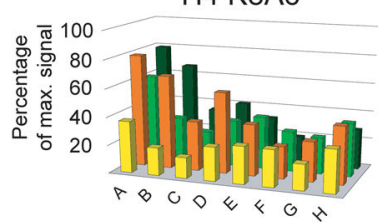

[1 shCDK8
FOS

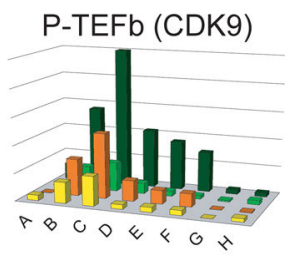

FACT (SPT16)

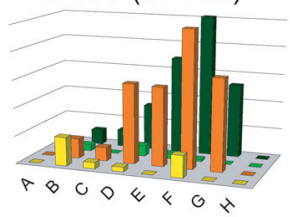

H4 K12AC

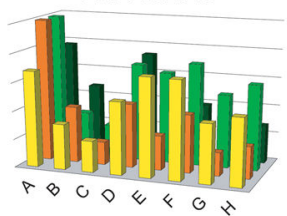

shCDK8
15 min serum

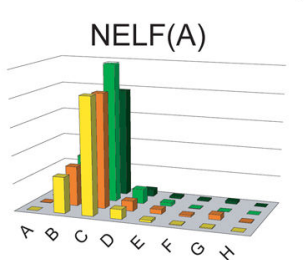

b EGR1

TFIIH (CDK7)
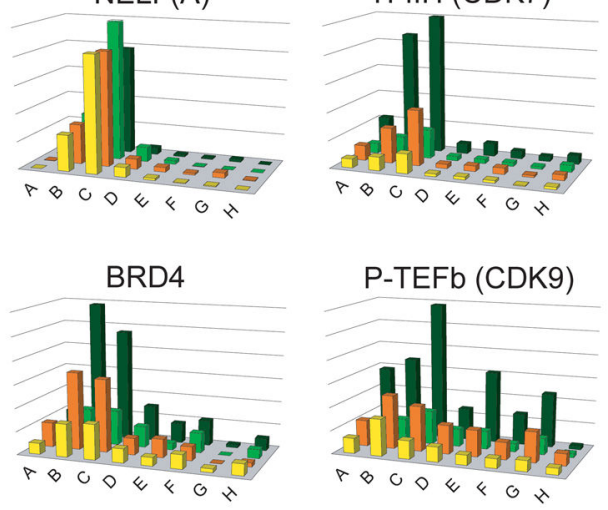

H3 K9Ac

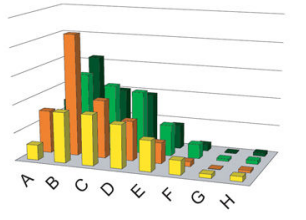

Control

0 min serum

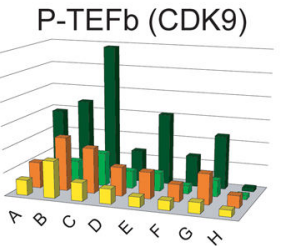

BRD4

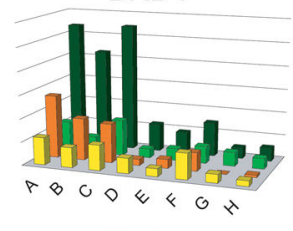

Control

15 min serum

Figure 5. CDK8 is required for recruitment of CDK7, CDK9 and BRD4 to IEGs

(a) ChIP for TFIIH (CDK7), P-TEFb (CDK9), NELF(A), DSIF (SPT5), FACT (SPT16), BRD4, histone $\mathrm{H} 4$ acetylated at lysines 8 and 12 (H4 K8Ac and K12 Ac), and histone H3 acetylated at lysine 9 (H3 K9Ac) at the FOS locus as described in Figure 2. (b) ChIP for TFIIH (CDK7), P-TEFb (CDK9) and BRD4 at the EGR1 locus as described in Figure 2. Average data from at least three independent experiments is represented. 
a

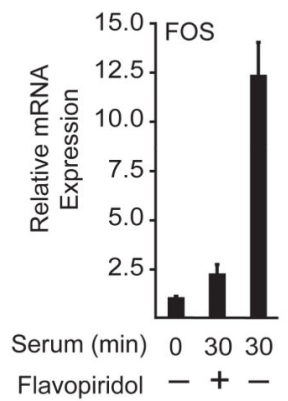

Q-RT-PCR

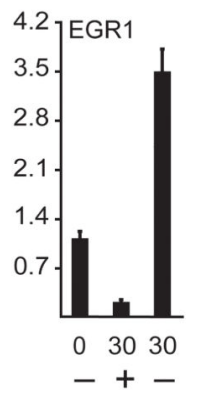

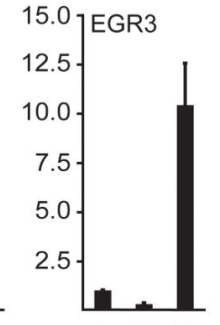

03030

$-+-$

b

EGR1

ChIP
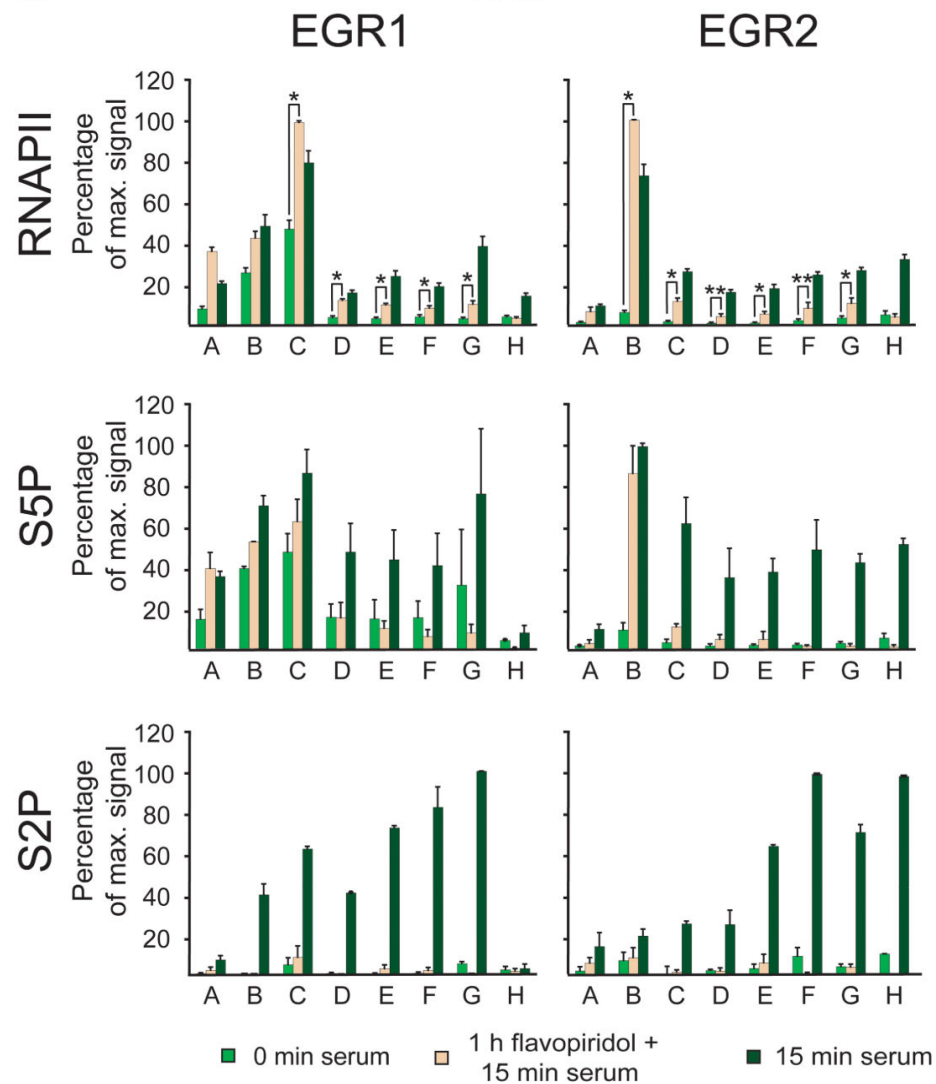

Figure 6. CDK9 inhibition mimics the post-recruitment defects in RNAPII activity observed in shCDK8 cells

(a) mRNA levels of FOS, EGR1, EGR2 and EGR3 were measured by Q-RT-PCR as in Figure 1, in serum starved cells treated with serum for 30 minutes with and without 1 hour of flavopiridol $(150 \mathrm{nM})$ pre-treatment. Data from at least three independent experiments is represented as mean -/+ standard error of the mean. (b) ChIP for RNAPII, S5P and S2P at the EGR1 and EGR2 loci following serum stimulation with or without flavopiridol (150 nM) pre-treatment. Average data from at least three independent experiments is represented. 

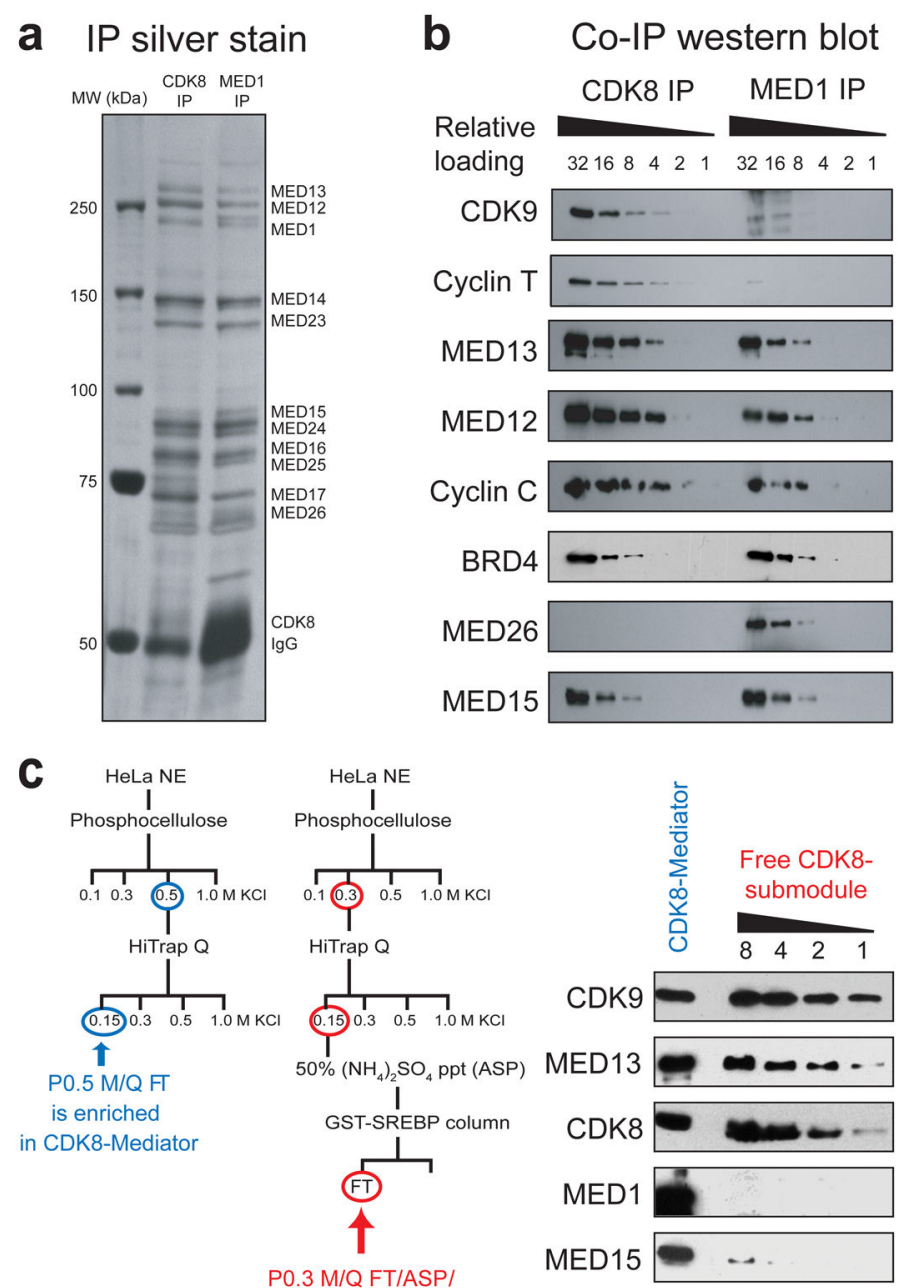

P0.3 M/Q FT/ASP / SREBP FT is enriched in free CDK8-submodule

Figure 7. P-TEFb associates with CDK8-Mediator

(a) Silver stain of CDK8 and MED1 immunoprecipitates (IPs). Mediator and associated cofactors were immunopreciptated from Hela nuclear lysates using antibodies specific for CDK8 (CDK8-Mediator and CDK8-submodule) and MED1 (core Mediator and CDK8Mediator). (b) Quantitative immunoblotting of IP elutions show CDK9 and cyclin T1 enriched in the CDK8 IP. c, Immunoblotting purified fractions of CDK8-Mediator and CDK8-submodule shows that CDK9 is detected in both the CDK8-submodule enriched (P0.3M/QFT/ASP/SREBP FT) and CDK8-Mediator enriched (P0.5M/QFT) fractions. 